\title{
Introduction
}

\section{Introduction to Thematic Clinical Issue of Neuropsychopharmacology in Late-Life Depression}

\author{
Charles F Reynolds III*, I,
'Psychiatry, Neurology, and Neuroscience, University of Pittsburgh School of Medicine, Pittsburgh, PA, USA; ${ }^{2}$ Intervention Research Center for Late
Life Mood Disorders, WPIC, USA
}

Neuropsychopharmacology (2004) 29, 2225, advance online publication, 7 October 2004; doi: I 0. I 038/sj.npp. I 300566

The series of manuscripts published in this issue of Neuropsychopharmacology underscore some of the challenges in understanding, and improving, treatment response in late-life major depression. A fundamental issue raised by these papers, in the aggregate, has to do with the neurobiological substrates of depression in old age and how these determine the characteristics of treatment response. Is depression in old age really cerebrovascular disease or Alzheimer's dementia? How should we (re-) conceptualize the goals of treatment, given the new data in these papers? These are old questions, but their perennial importance is highlighted by this issue of the journal.

Taylor and Doraiswamy review the evidence base that acute antidepressant pharmacotherapy is effective in old age to bring about response, but as Taylor et al also highlight, the definition of 'response' and 'remission' needs further consideration in relation to predicting longer-term treatment response variability. What is new in the past decade is the emerging consensus that old-age depression should be treated to the lowest possible degree of depressive symptomatology to minimize risk of later relapse. Partial response or $50 \%$ reduction in depressive symptoms inventories just is not good enough anymore. The challenge now confronting clinical researchers and clinicians alike, however, is the optimal sequence of treatments to get to this result, because typically first-line treatment suffices in only about half of older patients to achieve response and remission.

The manuscripts by Potter et al and by Alexopoulos et al highlight some of the progress being made in the understanding of treatment response modifiers in late-life

\footnotetext{
This work was supported in part by P30 MH52247, R37MH43832, and $\mathrm{ROI} \mathrm{MH} 37869$.

*Correspondence: Dr CF Reynolds III, Psychiatry, Neurology, and Neuroscience, University of Pittsburgh School of Medicine, Pittsburgh, PA, USA, Tel: + 412246 64l4, E-mail: Reynoldsc@@upmc.edu

Received 5 August 2004; accepted 6 August 2004
}

depression. Both papers signal the importance of executive dysfunction in compromising treatment response, a theme also struck in the spectroscopic data of Elderkin-Thompson and co-workers. What is new in the past decade is the emerging consensus that cognitive impairment is prevalent, pleomorphic, and persistent in late-life depression. The neurobiological and treatment response implications of this observation are just beginning to be unraveled, however.

Smith et al and Meltzer et al underscore the importance of serotonin transporter polymorphisms and serotonin $1 \mathrm{~A}$ receptor binding for explaining treatment response variability in old-age depression. Dysfunction in 5HT1A autoreceptor activity, as observed in the brainstem region of dorsal raphe nucleus, may play a central role in the mechanisms underlying treatment response to SSRIs in latelife depression. Similarly, Sheline and co-workers report decreased hippocampal 5HT $2 \mathrm{~A}$ receptor binding in older depressed patients, without significant differences between early- vs late-onset depression.

Finally, the papers published here raise the question of the relationship between depression in later life and dementia. In one of the first studies of its kind, Sweet et al report that neuropathologic changes of Alzheimer's dementia were predominant, occurring with greater frequency than cerebrovascular or Lewy Body changes, in late life depressed subjects who went on to develop dementia.

Taken together, these contributions from investigators in geriatric psychiatry point to the need to reconceptualize the goals of depression treatment in old age. If cognitive impairment is such a central but neglected aspect of treatment studies, for example, how should we formulate new treatment studies designed to get patients all the way well and keep them well, preserving as long as possible intact cognitive functioning? The availability of new tools to inform intervention and prevention strategies, as illustrated by papers in this issue of NPP, hails a new era in our search for ways to decrease a major source of illness-related disability in later life. 